\title{
Risk factors for the development of Horner's syndrome following interscalene brachial plexus block using ropivacaine for shoulder arthroscopy: a randomised trial
}

\author{
Michał J. Stasiowski ${ }^{1}$, Marek Zuber ${ }^{2}$, Radosław Marciniak ${ }^{1}$ Michał Kolny ${ }^{2}$, Ewa Chabierska ${ }^{2}$, \\ Przemysław Jałowiecki ${ }^{1}$, Aleksandra Pluta ${ }^{1}$, Anna Missir ${ }^{1}$ \\ ${ }^{1}$ Department of Anaesthesiology and Intensive Care, School of Medicine with the Division of Dentistry \\ in Zabrze, Medical University of Silesia, Katowice, Poland, St. Barbara's Memorial Hospital WSS no. 5 \\ Trauma Centre, Sosnowiec, Poland \\ ${ }^{2}$ Department of Anaesthesia, Endoscopic Surgery Clinic 'Sport-Klinika', Żory, Poland
}

\begin{abstract}
Background: Horner's syndrome is comprised of a set of symptoms caused by a permanent or transient ipsilateral sympathetic trunk lesion or paralysis. It may occur after numerous pathologies in the cervical region, epidural, spinal anaesthesia, and interscalene, transscalene, supraclavicular, or infraclavicular brachial plexus block. The aim of this randomised, prospective clinical study was to evaluate the effect of the interscalene brachial plexus block (IBPB) technique on the occurrence rate of Horner's syndrome and identify contributing risk factors.

Methods: 108 randomly selected patients of ASA I-III status were scheduled for elective shoulder arthroscopy. The patients received $20 \mathrm{~mL}$ of $0.5 \%$ ropivacaine either with ultrasound (US)-guided IBPB (U), peripheral nerve stimulation (PNS)-confirmation IBPB (N), or US-guided, PNS-confirmed IBPB (dual guidance; NU).

Results: We observed that Horner's syndrome developed in $12 \%$ of the $N$ group, $6 \%$ of the NU group, and $9 \%$ of the $\mathrm{U}$ group. The differences in the rates were not statistically significant $(P=0.616)$. Regardless of the technique used to induce IBPB, our study did not demonstrate any particular anthropometric parameter that predisposed the patients to the development of Horner's syndrome. Interestingly, our results showed that NU patients with Horner's syndrome were significantly younger than NU patients without Horner's syndrome.

Conclusion: The precision of IBPB by use of the dual guidance technique may reduce the rate of Horner's syndrome. The higher water concentration in the prevertebral spaces of younger patients may create better conditions for the diffusion of ropivacaine, which may result in a statistically significant higher HS rate.
\end{abstract}

Anaesthesiology Intensive Therapy 2018, vol. 50, no 3, 215-220

Key words: brachial plexus block, intrascalene; Horner's syndrome; arthroscopy; local anaesthetics, ropivacaine

Horner's syndrome (HS) is comprised of a set of symptoms caused by a permanent or transient ipsilateral sympathetic trunk lesion or paralysis. It is characterized by miosis, ptosis, and anhidrosis, with or without enophthalmia. It may occur after a number of pathologies associated with the cervical region [1-7] or epidural [8], spinal anesthesia [9], as well as combined spinal-epidural anesthesia [10]. Despite the different techniques for blocking brachial plexus performed by the interscalene [11], transscalene [12], and sometimes, supraclavicular [13, 14], and infraclavicular [15] local anaesthetics (LA), could still diffuse via the prevertebral spaces, block sympathetic nerves in the cervical region, as well as also spread to the sympathetic ganglia (the stellate ganglion), and could cause transient characteristic symptoms [16].

To our knowledge, no study has been conducted to evaluate the influence of interscalene brachial plexus block (IBPB) techniques on the rate of development of HS. In ad- 
dition, no anthropometric risk factors for the development of HS after brachial plexus block have been established. Therefore, we aimed to identify if any particular technique of IBPB may reduce the risk of occurrence of HS. Moreover, we decided to search for anthropometric risk factors of the above-mentioned symptom in order to identify a group of patients who may be predisposed to its occurrence due to their potentially specific anthropometry, as HS may influence their satisfaction with regional anaesthesia (RA).

\section{METHODS}

Our prospective, randomised, clinical study was approved by the Bioethics Committee of the Silesian University of Medicine in Katowice. A total of 108 patients were enrolled in the study. Data were obtained from patients who underwent elective shoulder arthroscopy and were classified as American Society of Anesthesiologists (ASA) grades I-III. The exclusion criteria were as follows: neurological deficit in the upper arm; allergies to amide LAs; coagulopathy; pregnancy; and the withdrawal of formerly given written consent.

After written informed consent was obtained, the patients were randomly allocated using the sealed envelopes method to one of three groups that were categorized by different IBPB techniques: ultrasound (US)-guidance IBPB (group U); peripheral nerve stimulation (PNS)-confirmation IBPB (group N); or US-guided with PNS-confirmed IBPB (dual guidance; group NU). All blocks were performed using $20 \mathrm{~mL}$ of $0.5 \%$ ropivacaine (Ropimol, Molteni Farmaceutici, Scandicci, Italy). After the block was performed, duration, onset time, and block effectiveness rated with the modified Lovett rating scale (LRS) were studied. An insufficient block was resolved by conversion to general anaesthesia.

On the morning of the day of surgery, patients were premedicated with $7.5 \mathrm{mg}$ oral midazolam. In the operating room, venous access was placed and $500 \mathrm{~mL}$ of crystalloid was infused. To improve comfort during regional anaesthesia, all patients received $0.1 \mathrm{mg}$ of intravenous fentanyl. The patients' heart rates, haemoglobin oxygen saturation, noninvasive systolic diastolic and mean arterial pressures, as well as cardiac rhythm were continuously monitored using a multiparameter anaesthetic monitor. Data were recorded with a 5-min sampling time in the anaesthetic protocol.

In the $\mathrm{N}$ group, all blocks were performed using Meier's modification (needle insertion point was at the level of the thyroid cartilage) while the needle was directed at a $30^{\circ}$ angle towards the middle part of the clavicle. Compared with traditional Winnie's access, Meier's modification is known to be as safe, but more efficient [17]. The procedure was begun with an exam that consisted of palpating the scalene muscles in the medial neck triangle and the interscalene groove lying between them. The time it took to complete the palpation was included in the regional block time. After skin sterilisation, local anaesthetic was administered at a dose of $1 \mathrm{~mL}$ of $1 \%$ lidocaine solution. IBPB was performed using a Contiplex D set (Contiplex D, B. Braun Inc., Melsungen, Germany) connected to a nerve stimulator (Stimuplex HNS12, B. Braun Inc., Melsungen, Germany). The stimulator settings were as follows: current, $0.3 \mathrm{~mA}$; impulse time duration, $0.1 \mathrm{~ms}$; and impulse frequency, $1 \mathrm{MHz}$. Motor response of either the pectoral muscles, triceps, or biceps brachii was considered to indicate proper needle placement. Subsequently, $20 \mathrm{~mL}$ of $0.5 \%$ ropivacaine was administered. A catheter was placed in the region of the anesthetised brachial plexus (BP) for postoperative pain treatment with a continuous infusion of $0.2 \%$ ropivacaine.

In the $U$ group, BP roots were visualised using an ultrasound system (Sonosite M-Turbo, Sonosite, Bothell, USA) equipped with a 13-MHz linear probe (HFL38/13-6 MHz, Sonosite, Bothell, USA). The procedure was initiated with a precise ultrasonographic scan of the lateral neck triangle. Examination time was included in the IBPB time. After the skin was sterilised, local anaesthesia was administered using $1 \mathrm{~mL}$ of a $1 \%$ lidocaine solution. The ultrasound transducer was covered using a sterile cover (Safersonic Conti, Safersonic, Ybbs, Austria) and was used together with a sterile hypoallergenic transmission gel (Aquasonic 100, Parker, Fairfield, USA). IBPB was performed using a Contiplex D set (Contiplex D, B. Braun Inc., Melsungen, Germany) by applying an out-of-plane technique similar to that used in group N. Ultrasound guidance was used to ensure proper needle placement. This was followed by the administration of $20 \mathrm{~mL}$ of $0.5 \%$ ropivacaine. The spread of the LA around the nerve roots was observed on the ultrasound monitor. Subsequently, a catheter was placed for continuous infusion of $0.2 \%$ ropivacaine.

In the NU group, although IBPB was performed in a manner similar to that of group $U$, the needle was attached to a PNS set in the same manner that is described in group N. In this group, the placement of the needle was confirmed two ways: visually and by electrostimulation (dual guidance). IBPB time was calculated until the end of the $0.5 \%$ ropivacaine injection. The clocked time for complete administration of LA was inclusive of the time it took to place the catheter and the sterile catheter dressing.

All blocks were performed by experienced anaesthesiologists skilled in both techniques (over 5 years in this field). After the block was performed, onset times, duration and IBPB's effectiveness were assessed by experienced anaesthesiologists skilled in both techniques (over 5 years in this field) who did not perform the IBPBs in this study and were blinded to the patients' group allocations. Sensory block examinations were performed at 5-min intervals by using ethanol-sprayed woollen swabs and pin pricks with 
a 22-gauge (G) needle. Motor block examinations were performed according to the modified LRS (6: normal muscular force; 5: slightly reduced muscular force; 4 : pronounced reduction of muscular force; 3 : slightly impaired mobility; 2: pronounced mobility impairment; 1 : almost complete paralysis; and 0: complete paralysis) and served as an indicator of surgical readiness. IBPB duration was designated as the time interval starting from a satisfactory sensory block until the very first pain perception requiring infusion of LA via the catheter placed in the region of the BP roots.

If there were signs of pain perception during surgery, conversion to general anaesthesia was administered. These cases were labelled as incomplete blocks. In the operating room, anaesthesiologists, who were not involved in the study, administered the appropriate sedation to patients either by continuous intravenous infusion of propofol or single doses of intravenous midazolam to improve their comfort during operation.

After IBPB was performed, the patients were observed for the development of Horner's syndrome (meiosis, ptosis, enophthalmia, anhidrosis, and conjunctival hyperaemia): before the surgery was started, after discharge to postoperative unit, every two hours in postoperative unit and, finally, at the first demand for postoperative analgesia. If at least meiosis, ptosis and enophthalmia were present in a patient after IBPB at any stage of the perioperative period, this case was labelled as HS.

\section{STATISTICS}

All analyzed groups were assessed using the ShapiroWilk test for estimating normal distributions. As the distributions were not normal, the non-parametric Mann-Whitney $U$ test was used for statistical analysis. Statistical significance was set at $P<0.05$. All calculations were made using Statistica 12.0 (StatSoft, Inc. USA).

\section{RESULTS}

Among all the patients who were scheduled for IBPB, there was no statistically significant difference in gender, height, body mass or duration of surgery.

The number of patients who did not require conversion to general anaesthesia was $80.43 \%$ of the patients in group NU, $75.56 \%$ of group $U$, and $41.46 \%$ of group $N$. Patients receiving IBPB with $20 \mathrm{ml}$ of $0.5 \%$ ropivacaine with the assistance of US visualisation and PNS-confirmation for brachial plexus identification had a reduced rate of onset of HS (6\% of the NU group) compared with $12 \%$ of the $\mathrm{N}$ group. Our results also show that refraining from using PNS assistance led to an increase rate of $\mathrm{HS}$ as seen in Table 1.

Regardless of the technique of IBPB used, globally, our study did not demonstrate any particular anthropometric parameter predisposing patients to develop HS (see Table 2).

\section{GROUP N}

As shown in Table 3, there were no statistically significant differences in the anthropometric parameters between those patients in group $\mathrm{N}$ who developed HS (HS-N) and those who did not develop HS (nHS-N).

\section{GROUP U}

As shown in Table 3, our data shows that we could not find any statistically significant differences in the anthropometric parameters between patients who developed HS (HS-U) and those who did not develop HS (nHS-U).

\section{GROUPNU}

As shown in Table 3, there were no statistically significant differences in most of the anthropometric parameters between those patients in group NU who developed HS (HS-NU) and those who did not develop HS (nHS-NU). However, we found an interesting difference between the patients' ages. HS-NU patients appeared to be younger to a statistically

Table 1. Rate of Horner's syndrome (HS) following interscalene brachial plexus block (IBPB) in studied groups

\begin{tabular}{lccc}
\hline Group & HS rate (\%) & Gender & P-value \\
\hline $\mathrm{NU}(\mathrm{n}=34)$ & $2(6 \%)$ & $\begin{array}{c}\text { Male 70\%, } \\
\text { Female 30\% }\end{array}$ & \\
$\mathrm{U}(\mathrm{n}=34)$ & $3(9 \%)$ & $\begin{array}{c}\text { Male 75\%, } \\
\text { Female 25\% }\end{array}$ & 0.616 \\
$\mathrm{~N}(\mathrm{n}=40)$ & $5(12 \%)$ & Male $80 \%$, & \\
& & Female 20\% & \\
\hline
\end{tabular}

Table 2. Global anthropometric parameters of patients manifesting Horner's syndrome (HS) versus not manifesting Horner's syndrome ( $n$-HS) receiving interscalene brachial plexus block (IBPB), regardless of group allocation

\begin{tabular}{|c|c|c|c|c|c|c|c|c|}
\hline \multirow{3}{*}{$P$-value } & \multicolumn{2}{|c|}{ Height (cm) } & \multicolumn{2}{|c|}{ Body mass (kg) } & \multicolumn{2}{|c|}{ Age (yrs) } & \multicolumn{2}{|c|}{ BMI $\left(\mathrm{kg} \mathrm{m}^{-2}\right)$} \\
\hline & \multicolumn{2}{|c|}{0.163} & \multicolumn{2}{|c|}{0.578} & \multicolumn{2}{|c|}{0.713} & \multicolumn{2}{|c|}{0.219} \\
\hline & Mean & SD & Mean & SD & Mean & SD & Mean & SD \\
\hline HS & 172.00 & 9.02 & 79.00 & 23.39 & 26.28 & 5.81 & 26.45 & 13.29 \\
\hline$n-H S$ & 174.90 & 8.30 & 79.86 & 14.49 & 26.11 & 4.77 & 25.63 & 15.22 \\
\hline
\end{tabular}


Table 3. Anthropometric parameters of patients manifesting Horner's syndrome (HS) versus not manifesting Horner's syndrome (n-HS) receiving interscalene brachial plexus block (IBPB), according to group allocation by US-guidance IBPB (U), peripheral nerve stimulation-confirmation IBPB (N), or US-guided, PNS-confirmed IBPB (dual guidance; NU)

\begin{tabular}{|c|c|c|c|c|c|c|c|c|c|}
\hline & & \multicolumn{2}{|c|}{ Height (cm) } & \multicolumn{2}{|c|}{ Body mass (kg) } & \multicolumn{2}{|c|}{ Age (yrs) } & \multicolumn{2}{|c|}{$\mathrm{BMI}\left(\mathrm{kg} \mathrm{m}^{-2}\right)$} \\
\hline & & Mean & SD & Mean & SD & Mean & SD & Mean & SD \\
\hline \multirow[t]{3}{*}{$\mathrm{N}$} & $\mathrm{HS}$ & 174.4 & 11.76 & 87.2 & 29.41 & 38.6 & 12.7 & 28.12 & 7.35 \\
\hline & $\mathrm{n}-\mathrm{HS}$ & 175.81 & 8.36 & 80.34 & 11.62 & 41 & 13.23 & 25.91 & 2.7 \\
\hline & $P$-value & \multicolumn{2}{|c|}{0.721} & \multicolumn{2}{|c|}{0.841} & \multicolumn{2}{|c|}{0.902} & \multicolumn{2}{|c|}{0.876} \\
\hline \multirow[t]{3}{*}{$U$} & $\mathrm{HS}$ & 169 & 7.39 & 74.5 & 20.94 & 38.25 & 16.46 & 25.71 & 5.16 \\
\hline & $\mathrm{n}-\mathrm{HS}$ & 175.11 & 7.44 & 80.16 & 17.74 & 41 & 15.93 & 26.21 & 6.59 \\
\hline & $P$-value & \multicolumn{2}{|c|}{0.151} & \multicolumn{2}{|c|}{0.758} & \multicolumn{2}{|c|}{0.834} & \multicolumn{2}{|c|}{0.982} \\
\hline \multirow[t]{3}{*}{ NU } & HS & 172 & 5.66 & 67.5 & 3.54 & 26.67 & 7.64 & 22.81 & 0.31 \\
\hline & $\mathrm{n}-\mathrm{HS}$ & 173.86 & 9.17 & 79.11 & 13.41 & 43.46 & 14.94 & 26.18 & 4.04 \\
\hline & $P$-value & \multicolumn{2}{|c|}{0.485} & \multicolumn{2}{|c|}{0.148} & \multicolumn{2}{|c|}{0.048} & \multicolumn{2}{|c|}{0.178} \\
\hline
\end{tabular}

significant degree in comparison with nHS-NU patients (see Table 3).

\section{DISCUSSION}

Based on data from the current literature, the development of Horner's syndrome after a brachial plexus block occurs at a rate ranging from $4-37.5 \%$, depending on the technique and approach of regional anaesthesia, as well as the volume and dilution of LA used in these studies.

Jandard et al. [18] performed neurostimulation-guided single injections of $40 \mathrm{~mL}$ of $1.5 \%$ mepivacaine by the vertical lateral paracoracoid approach to create infraclavicular brachial plexus blocks for surgical procedures distal to the elbow. They observed HS in 4\% of the 100 patients included in their study. In a prospective clinical study comprised of 175 patients who received neurostimulation-guided infraclavicular vertical brachial plexus blockade with 400 $\mathrm{mg}$ of $1 \%$ Prilocaine and $50 \mathrm{mg}$ of $0.5 \%$ bupivacaine given as a single injection, Kilka et al. [19] observed 12 cases of Horner's syndrome. This is somehow contrary to the research of Rodríguez et al. [16] who found that large volume of LA solution $(46 \mathrm{~mL})$ remained below the clavicle and there was no HS in this group of 6 patients. Zaragoza-Lemus et al. [20] reported that Horner's syndrome developed in $8 \%$ of 50 shoulder surgery candidates, who received an interscalene block with a volume of $30 \mathrm{~mL}$ of $L A$ using the neurostimulation technique. In a prospective, randomised comparison between ultrasound-guided supraclavicular, infraclavicular, and axillary brachial plexus blocks, Tran et al. [21] demonstrated the development of HS at rates of $37.5 \%, 0 \%$, and $5 \%$, respectively.

In our study, we found that the dual guidance technique of IBPB lead to a reduction in the rate of development of HS $(n=2,6 \%)$ compared with the ultrasound $(U)$ technique $(\mathrm{n}=3,9 \%)$ and neurostimulation (NS) technique ( $\mathrm{n}=5,12$ $\%)$ although the difference was not statistically significant $(P=0.616)$.SinceHSoccurred with highervolumes ofanaesthetic, as seen in the studies by Jandard et al. [18] and Kilka et al. [19] we conclude that a reduction of $L A$ volume to $20 \mathrm{~mL}$, accompanied with a higher precision of LA administration may reduce the rate of $\mathrm{HS}$. We propose that the reason of this reduction in $\mathrm{HS}$ due to precision in LA administration may be explained by our comparison of NU and N groups. As the majority of LA solution in group NU (US visualized, PNS-confirmed) was injected closer to nerve roots, there may have been less chance for infiltrating the prevertebral spaces. In turn, there may have been less diffusion of LA particles to the sympathetic nerves in the cervical region and to the stellate ganglion. This is in contrast to the $\mathrm{N}$ group where LA solution could have been administered farther from the brachial plexus, thus also contributing to a higher rate of $\mathrm{HS}$, despite the lack of statistically significant differences.

Nevertheless, the main limitation of the study may be the fact that it could be underpowered, as HS was developed in relatively few patients. Therefore, further studies including much larger number of patients should be carried out in order to investigate the problem in detail.

We also showed that within the NU group, those who developed HS were significantly younger than those who did not. This finding is consistent with a report by TraiqAlzahrani et al. [17] They presented a case of delayed HS in a healthy 29-year-old male patient after he underwent ultrasound-guided catheter placement for post-operational analgesia of the brachial plexus after major open shoulder surgery. The patient developed HS on the ipsilateral side. The symptoms were typical: meiosis, ptosis, enophthalmia, anhidrosis, and conjunctival hyperaemia, and which started 1 hour after receiving $10 \mathrm{~mL}$ of $0.2 \%$ bupivacaine, which was 
administered through an interscalene catheter by the oncall team. The symptoms resolved after approximately $2 \mathrm{~h}$. On the other hand, Ilfeld et al. [22] observed no incidences of HS when they compared different techniques of $0.2 \%$ ropivacaine infusion for brachial plexus analgesia via an infraclavicular perineural catheter, at a rate of $12 \mathrm{~mL} \mathrm{~h}^{-1}$.

Our observations could be explained by the fact that younger patients have a higher water concentration in their tissues than older patients. LAs are composed of hydrophilic and lipophilic chains - thus as the tissue of the fascia layers has a higher water concentration, LA diffuses slower. According to an analysis by Balcerkiewicz et al. [23], bupivacaine is 1000 times more lipophilic than procaine, whereas ropivacaine is only 400 times more lipophilic. Therefore, in younger patients, who have a higher concentration of water in their tissues compared with the older patients, it is possible that the reason they developed HS more often was because the LA particles that were not absorbed by the lipophilic nerve roots automatically diffused through the prevertebral spaces to the sympathetic nerves and the stellate ganglion. In our opinion, fewer particles of ropivacaine, which is more hydrophilic, can diffuse through the prevertebral spaces of elderly patients because they have a lower water concentration. This may explain the lower rate of HS in the older patients of the NU group. The delayed onset of HS symptoms observed by Traiq-Alzahrani et al. [17], who used bupivacaine, may be because the initial particles of lipophilic bupivacaine were quickly absorbed by nerve roots, while the remaining particles of LA slowly diffused through the prevertebral spaces to below the clavicle causing HS 1 hour later. The study by Ilfeld et al. [22] used a $0.2 \%$ ropivacaine solution. In comparison with bupivacaine, which is a mixture of L- and S-isomers, ropivacaine is composed of only an Lisomer and is more hydrophilic. Therefore, it did not cause $\mathrm{HS}$ as it did not diffuse through the prevertebral spaces as easily as the lipophilic bupivacaine. In addition, a concentration of $0.2 \%$ ropivacaine does not reach adequate levels to diffuse via tissues in order to block the sympathetic chain and cause HS.

The observations above may be consistent with the findings of Sivashanmugam et al. [24] who compared the effects of an ultrasound-guided injection of $25 \mathrm{~mL}$ of $0.5 \%$ bupivacaine deep to the brachial plexus sheath at the supraclavicular fossa with an injection superficial to the sheath. They observed a faster onset of surgical anaesthesia and prolonged duration of postoperative analgesia in patients who received the block deep to the brachial plexus sheath. In their study, the need for diffusion via the superficial sheath strongly correlates with the delayed onset of motor and sensory IBPB.

In our opinion, the development of adverse events, such as HS and diaphragm paresis, strongly correlates with the amount of free LA particles diffusing to the target site. This results in either the expected IBPB or unwelcomed adverse effects.

In conclusion, the development of HS may depend mainly on the lipophilic potential, volume and dilution of $L A$ solution used to perform regional block, as well as the IBPB technique. The precision of IBPB by use of the dual guidance technique may reduce the rate of HS. The higher water concentration in the prevertebral spaces of younger patients may create better conditions for the diffusion of ropivacaine, which may result in a statistically significant higher HS rate. Nevertheless, patients perceive HS as an unwelcomed adverse effect that causes anxiety and discomfort. Regardless of which IBPB technique is used or the volume and dilution of LA administered, patients should be prepared for the possibility of developing HS and reassured that it is a transient condition.

\section{ACKNOWLEDGEMENTS}

1. Source of funding: none.

2. Conflict of interest: none.

\section{References:}

1. Kal A, Ercan ZE, Duman E, et al. Abducens nerve palsy and ipsilateral horner syndrome in a patient with carotid-cavernous fistula. J Craniofac Surg. 2015; 26(7): e653-e655, doi: 10.1097/SCS.0000000000002131, indexed in Pubmed: 26468854.

2. Fang $X, X u Z q$, Luo By, et al. Carotid artery dissection with Horner syndrome as main manifestation: a case report. Zhejiang Da Xue Xue Bao Yi Xue Ban. 2015; 44(2): 229-232, indexed in Pubmed: 26038145.

3. Padaki AS, Fitch RW, Stack LB, et al. Horner's syndrome after scalene block and carotid dissection. J Emerg Med. 2016; 50(5): e215-e218, doi: 10.1016/j.jemermed.2016.01.027, indexed in Pubmed: 26899514.

4. Lin YC, Chuang MT, Hsu CH, et al. First rib fracture resulting in horner's syndrome. J Emerg Med. 2015; 49(6): 868-870, doi: 10.1016/j. jemermed.2015.07.031, indexed in Pubmed: 26375810.

5. Meng K, Tian W, Lv Z, et al. Horner's syndrome subsequent to minimally invasive video-assisted thyroidectomy in two patients. Oncol Lett. 2015; 10(1): 459-462, doi: 10.3892/ol.2015.3159, indexed in Pubmed: 26171051.

6. Ginos J, Mcnally S, Cortez M, et al. Vertebral artery dissection and cord infarction - an uncommon cause of brown-séquard and horner syndromes. Cureus. 2015; 7(8): e308, doi: 10.7759/cureus.308, indexed in Pubmed: 26430582.

7. Foss-Skiftesvik J, Hougaard MG, Larsen VA, et al. Clinical Reasoning: Partial Horner syndrome and upper right limb symptoms following chiropractic manipulation. Neurology. 2015; 84(21): e175-e180, doi: 10.1212/WNL.0000000000001616, indexed in Pubmed: 26009566.

8. Kumar A, Battit GE, Froese AB, et al. Bilateral cervical and thoracic epidural blockade complicating interscalene brachial plexus block: report of two cases. Anesthesiology. 1971; 35(6): 650-652, indexed in Pubmed: 5124750

9. Dutton RP, Eckhardt WF, Sunder N. Total spinal anesthesia after interscalene blockade of the brachial plexus. Anesthesiology. 1994; 80(4): 939-941, indexed in Pubmed: 8024148.

10. Karaca Ö, Kumaş Solak S, Demirgan S, et al. [Horner syndrome following combined spinal-epidural anesthesia]. Agri. 2016; 28(3): 155-157, indexed in Pubmed: 27813034.

11. Urmey WF, Talts KH, Sharrock NE. One hundred percent incidence of hemidiaphragmatic paresis associated with interscalene brachial plexus anesthesia as diagnosed by ultrasonography. Anesth Analg. 1991; 72(4): 498-503, indexed in Pubmed: 2006740.

12. Nguyen HC, Fath E, Wirtz S, et al. Transscalene brachial plexus block a new posterolateral approach for brachial plexus block. Anesth Analg. 
2007; 105(3): 872-875, doi: 10.1213/01 ane.0000271916.26357.8d, indexed in Pubmed: 17717253.

13. Mak PH, Irwin MG, Ooi CG, et al. Incidence of diaphragmatic paralysis following supraclavicular brachial plexus block and its effect on pulmonary function. Anaesthesia. 2001; 56(4): 352-356, indexed in Pubmed: 11284823.

14. Neal JM, Moore JM, Kopacz DJ, et al. Quantitative analysis of respiratory, motor, and sensory function after supraclavicular block. Anesth Analg. 1998; 86(6): 1239-1244, indexed in Pubmed: 9620512.

15. Stav A, Reytman L, Stav MY, et al. Comparison of the supraclavicular, infraclavicular and axillary approaches for ultrasound-guided brachial plexus block for surgical anesthesia. Rambam Maimonides Med J. 2016; 7(2), doi: 10.5041/RMMJ.10240, indexed in Pubmed: 27101216.

16. Rodríguez J, Bárcena M, Alvarez J. Restricted infraclavicular distribution of the local anesthetic solution after infraclavicular brachial plexus block. Reg Anesth Pain Med. 2003; 28(1): 33-36, doi: 10.1053/ rapm.2003.50022, indexed in Pubmed: 12567341.

17. Alzahrani T, Alnajjar M, Algarni AD, et al. Delayed Horner's syndrome following ultrasound-guided interscalene brachial plexus block. Saudi J Anaesth. 2014; 8(1): 121-123, doi: 10.4103/1658-354X.125972, indexed in Pubmed: 24665252.

18. Jandard C, Gentili ME, Girard F, et al. Infraclavicular block with lateral approach and nerve stimulation. Regional Anesthesia and Pain Medicine. 2002; 27(1): 37-42, doi: 10.1097/00115550-200201000-00008.

19. Kilka HG, Geiger P, Mehrkens HH. Die vertikale infraklavikulare Blockade des Plexus brachialis Eine neue Methode zur Anesthesie der oberen Extremitet. Der Anaesthesist. 1995; 44(5): 339-344, doi: 10.1007/ s001010050162.
20. Zaragoza-Lemus G, Limón-Muñoz M, García-Reyes W. Ultrasonographic assessment of hemidiaphragm paralysis secondary to interscalene block. Cir Cir. 2012; 80(4): 352-356, indexed in Pubmed: 23374383.

21. Tran DeQ, Russo G, Muñoz L, et al. A prospective, randomized comparison between ultrasound-guided supraclavicular, infraclavicular, and axillary brachial plexus blocks. Reg Anesth Pain Med. 2009; 34(4): 366-371, doi: 10.1097/AAP.0b013e3181ac7d18, indexed in Pubmed: 19574871.

22. Ilfeld BM, Enneking FK. Infraclavicular perineural local anesthetic infusion: a comparison of three dosing regimens for postoperativeanalgesia. Anesthesiology 2004; 100(2): 395-402.

23. Balcerkiewicz A. Terapia i leki. Farm Pol. 2010; 66(6): 415-424.

24. Sivashanmugam T, Ray S, Ravishankar M, et al. Randomized comparison of extrafascial versus subfascial injection of local anesthetic during ultrasound-guided supraclavicular brachial plexus block. Reg Anesth Pain Med. 2015; 40(4): 337-343, doi: 10.1097/AAP.0000000000000264, indexed in Pubmed: 26066385.

\section{Corresponding author:}

Michał J. Stasiowski, Ph.D., M.D.

Department of Anaesthesiology and Intensive Care

Medical University of Silesia

Plac Medyków 1

41-200 Sosnowiec, Poland

e-mail:mstasiowski.anest@gmail.com

Received: 20.11 .2016

Accepted: 25.05.2018 